\title{
VOLODYMYR OLEKSANDROVYCH DERKACH (to 70th birthday anniversary)
}

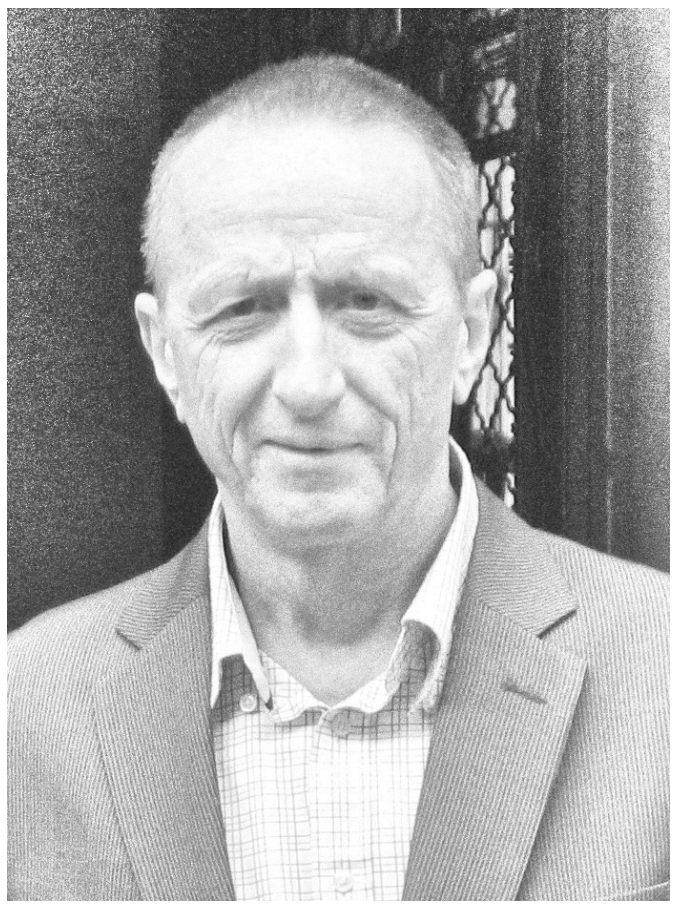

On December 12, 2020, an outstanding Ukrainian mathematician, Professor Volodymyr Oleksandrovych Derkach celebrated his 70th birthday anniversary.

He was born in a small city Chernigovka, Primorsky region of Russia to a family of a military and a pharmacist. In 1966 he graduated from high school in Donetsk and entered the Mathematics Faculty of Donetsk State University. Volodymyr started his research work during his students years under the supervision of E. R. Tsekanovskil. He developed extension theory of symmetric operators in rigged Pontryagin spaces. Being a postgraduate student he defined and studied an analogue of the Livsic characteristic function for unbounded quasi-selfadjoint operators in indefinite inner product spaces. V. Derkach defended his Candidate/PhD thesis "Spectral analysis of unbounded nonselfadjoint operators in indefinite inner product spaces" in 1978 at the Donetsk Institute of Applied Mathematics and Mechanics.

In 80s, the collaboration of V. Derkach and M. Malamud started. They developed the method of boundary triples (boundary value spaces) in the extension theory of symmetric operators in Hilbert spaces, a notion originating from the works of M.L. \& V.I. Gorbachuk, A.N. Kochubei, being also a specific type of reduction operator introduced earlier by J.W. Calkin. Together they introduced an important notion of the abstract Weyl function of a symmetric operator associated with a boundary triple and established its connection with the Kreı̆n's $Q$-function and Livsic characteristic function of a nonselfadjoint operator. Furthermore, in joint papers with E.R. Tsekanovskiǔ, accretive and sectorial extensions of a positive operator were characterized both in terms of the 
Weyl function and the characteristic function. In a series of joint papers V. Derkach and M. Malamud showed that the boundary triples technique provides a new approach to the theory of characteristic functions of non-selfadjoint operators. They applied this technique to different problems of extension theory, spectral problems for boundary value problems of differential operators, and to a large class of interpolation problems. They found a universal formula for resolvent matrices of symmetric operators and applied it to various moment problems of Hamburger and Stieltjes type. For a symmetric operator with several gaps the Kreın's problem concerning the description of selfadjoint extensions preserving these gaps was solved. These results have been summarized in their monograph "Extension Theory of Symmetric Operators and Boundary Value Problems" published in 2017 in the series of Proceedings of Institute of Mathematics of NAS of Ukraine.

In late $80 \mathrm{~s}, \mathrm{~V}$. Derkach started to work with the notions of Weyl functions and boundary value spaces of symmetric operators or linear relations in indefinite inner product spaces. His interest in this area with its various applications, like indefinite interpolation and moment problems, has continued actively until the present days. In particular, the Schur algorithm for scalar indefinite Hamburger and Stieltjes moment problem was elaborated in joint works with his PhD students M. Derevyagin and I. Kovalyov. Convergence of diagonal and subdiagonal Padé approximants for a wide class of generalized Nevanlinna and Stieltjes functions was studied. Some of these studies were included in his Doctor thesis "The methods of boundary values in the extension theory of symmetric operators in the spaces with indefinite metric" defended in 2003 at the Institute of Mathematics of NAS of Ukraine. Up to now the operator theory in Pontryagin and Krel̆n spaces with applications is one of the main topic for scientific interests of $\mathrm{V}$. Derkach and he is one of the leading experts in the world in this field.

During the last two decades V. Derkach has been actively collaborating with mathematicians from various countries. In a series of joint papers with S. Hassi (Finland) and H. de Snoo (the Netherlands) operator models for various subclasses of generalized Nevanlinna functions and singular finite rank perturbations of selfadjoint operators in Pontryagin spaces were constructed and investigated by the methods of boundary triple approach. On the other hand, jointly with M. Malamud they studied realization problems for general Nevanlinna families and introduced the notions of a boundary relation and an associated Weyl family, and then elaborated a geometric approach to Kreln formula for generalized resolvents with a coupling method. Various new classes of generalized boundary triples were introduced and characterized in purely analytic terms via their Weyl functions. In a fruitful collaboration with H. Dym (Israel) indefinite Schur-NevanlinnaPick type interpolation problems with degenerate Pick matrices were investigated and, for instance, functional models for symmetric operators in indefinite de Branges spaces have been studied. Very recently the problem of similarity of an indefinite Sturm-Liouville operator to a selfadjoint operator was investigated by the methods of extension theory jointly with B. Čurgus (USA) and C. Trunk (Germany).

Professor Derkach is the author of more than 80 articles published in leading mathematical journals. He is an excellent lecturer and teacher; he has been a supervisor of 6 $\mathrm{PhD}$ theses. His teaching activity is mainly connected with Vasyl' Stus Donetsk National University (former Donetsk State University), where he has been working more than 30 years.

In 2003-2012 he was the head of the Department of Mathematical Analysis and Function Theory in that university. In 2014 Donetsk was occupied by Russian armed forces and V. Derkach with Donetsk National University moved to Vinnytsia.

Throughout his long scientific activity Professor Derkach has worked at several universities in the Netherlands, Finland, USA, Israel, and Germany. He was honored with Weston Visiting Scholarship grant (Weizmann Institute of Science, Israel), the Fulbright Research 
grant (University of Massachusetts Lowell, USA) and DFG Mercator professorship grant (Technical University of Ilmenau, Germany). V. Derkach is a member of the American Mathematical Society and of the Editorial boards of Ukrainian Mathematical Bulletin and Carpathian Mathematical Publications.

We cordially greet Volodymyr Oleksandrovych with the jubilee and wish him good health, happiness and further creative achievements.

Editorial Board 